\title{
Tribological Aspect of Dynamic Deviations in Ring -Frame Twisting System due to Dynamic Malalignment of Spindle
}

\author{
Marina Michalak \\ Textile Department Technical University of Lodz, Lodz, Zeromskiego 116, Poland \\ Received Aug. 17,1999;Accepted for Publicati on Jan. 19,2001
}

\begin{abstract}
The paper presents a fragment of a research project aimed at defining the dynamics and tribology of the real twisting system of a ring spinning frame, i.e. a system with regular and/or random occurrence of faults in its operation. The object of study are the dynamic disturbances and their tribological consequences, which occur in the operation of the twisting system of a worsted spinning ring-frame due to faulty rotation of the spindle caused by play in its bearing. Studied was a twisting system provided with a taper ring (HZ) and ' $\mathrm{J}$ ' steel traveiler. The forces were determined, for a case of three-contact inter-action between the traveller and ring, which act on the traveller and yarn if the spindle axis deviates from the vertical so that its tip traces an ellipse or a circle. It is shown that this faulty operation of the twisting system has a destructive effect on the inter-action of the traveller-ring-yam (TRY) trio, which aggravates with time and has a deteriorating effect on the performance of the system
\end{abstract}

\section{Introduction}

The last operation in the worsted spinning of wool is the one which takes place in the twisting system of the ring frame and consists in simultaneous twisting and winding of the fibre stream coming from the drafting system. In the twisting system the yarn is rotating round the spindle axis, between the guide eye and traveller, and the centrifugal force makes it form a balloon or, in other words, a curved three-dimensional geometrical form. In the various segme nts of the yarn, between its points of contact with the guide eye, traveller, and package on the bobbin, the tension in it takes various values which are, however, lower than the breaking strength of the yarn. This pre-planned relation between the maximum tension in the yarn and its breaking strength is a function of speed of the spindle and weight of the traveller.

When analysing the dynamics of the twisting system of a ring spinning frame - used in the spinning of cotton or worsted spinning of wool - distinction has to be made between the two types of systems, i.e. systems whose dynamics is limited to the classical mechanics and systems in which certain random factors are also present. The first type are systems in which the operation of the component elements is regular in time and as designed and systems in which the operation of the component elements - due, for instance, to incorrect assembling or wear - is faulty but regular in time. In the latter case, faulty operation may be due to eccentric disposition of spindle axis with respect to the centre of the ring. For the purposes of analysis it is assumed, in either case, that the yarn is inextensible and its mass or weight is regularly distributed along its axis.

The second type are systems in which alongside of elements which operate ideally, or faulty, but in a regular manner, there is random occurrence of irregular operation, attributable either to the mechanical elements of the system or to the structure of the yarn. Whereas the first contingency can be accounted for by temporary changes in the position of the top part of the spindle axis (if there is play in spindle bearing), the second contingency is a consequence of the fact that the yarn is extensible and its mass is irregularly distributed along its axis, as is the case with some fancy yarns.

Decision, which of the two types is represented by the given twisting system, is not always simple. In the first instance, estimation is needed in advance of how intensively the dynamic condition of the twisting system is affected by the random factor or factors. However, one must be aware that in trying to assess the effect of a random factor on the operation of a dynamic system it is necessary to know the values of certain statistics which cha-racterise the effects of random factors. Whereas estimation of the effect of random distribution of yarn weight on the traveller is possible on the grounds of the known theories of distribution, estimation of the random temporary positions in space of the spindle top requires special empirical procedures to determine the relevant statistics.

Owing to the present world-scale collaboration of research centres, available is a mathematical formulation, based on the classical dynamics, of the dynamics of a faultless cotton-system ring-frame twisting system in which

* Corresponding author E-mail: marina@ck-sg.p.lodz.pl 
the conventional traveller operates in one-point contact with the ring [1-4]. On the basis of this formulation it is possible to pre-plan the optimal technological parameters such as: variability of the form of yarn balloon in the course of spinning; variation of tension in the yarn; and the corresponding reactions of the traveller to the ring. There is little doubt that the theory of faultless cotton-system ring-frame twisting system can be successfully applied to the worsted spinning ring-frame twisting system.

Dynamic deviations do also occur in the twisting system of the woollen-system ring frame, but since the principle of operation of this system is different from that of the worsted system, investigation of faulty operation of the woollen-system twisting system requires correspondingly different methods.

\section{The dynamics of a real twisting system}

\subsection{Current results}

The present paper covers a fragment of a larger project aimed at performing a detailed analysis of the dynamics and tribology of the friction trio traveller-ring-yarn, TRY, which constitutes the twisting system of a ring spinning frame and is often encumbered with faults that occur regularly and/or stochastically.

The project has been inspired by lack of a theoretical formulation of the problem in the relevant literature and is already a second project of this kind. In the earlier project [5-9], analysed and experimentally verified was a model of a ring-frame twisting system with a fault consisting in that the spindle axis was eccentrically disposed, by $\varepsilon>0$, to the centre of the ring. Analysis of the problem showed that the fault $\varepsilon>0$ introduced to the dynamic condition of the twisting system a periodic change occurring in cycles equal to one lap of the traveller and involving the speed of the traveller, tension in the ballooning yarn, variation of the form of the balloon, and reactions of the yarn and ring to the traveller, which became periodic quantities whose amplitudes of oscillation were, among others, functions of spindle speed $n$ and spindle eccentricity $\varepsilon$. It needs to be noted that at $\varepsilon>0$ the periodicity of the phenomena occurring in the twisting system influence the tribological conditions of the twisting system and, therefore, also the effects of interaction of the elements of TRY.

To describe a momentary condition of the studied TRY developed was an analytic-and-experimental model which was characteristic in that the traveller had a pre-assumed speed and, likewise, pre-assumed was the model of the momentary reaction $\mathrm{T}_{\mathrm{b}}$ of the ballooning yarn to the traveller. A second model was developed based on experimentally determined position, in space, of the vector $T_{b}$ by measuring momentary values of the angles $(\alpha, \beta, \gamma)$ which $\mathrm{T}_{\mathrm{b}}$ makes at $t$ $=0$ (during one lap of traveller) correspondingly with $(x, y, z)$ of a three-dimensional system of co-ordinates originating at point $O$ of traveller contact with the ring. The model was verified experimentally [10].

2.2. Dynamic and tribological aspect of deviations in the twisting system of a ring frame caused by 'whipping' of the spindle (due to spindle bearing play)

\subsubsection{Determinations and symbols}

Reported is analysis of the effect of spindle 'whipping' (in a worsted ring frame) due to spindle bearing play, i.e. of the effect of momentary changes in the position in space of the upper part of spindle axis, on the momentary dynamic condition of TRY composed of a taper ring (HZ), ' $J$ ' traveller, and yam which is assumed to be both inextensible and regular (Fig. 1).

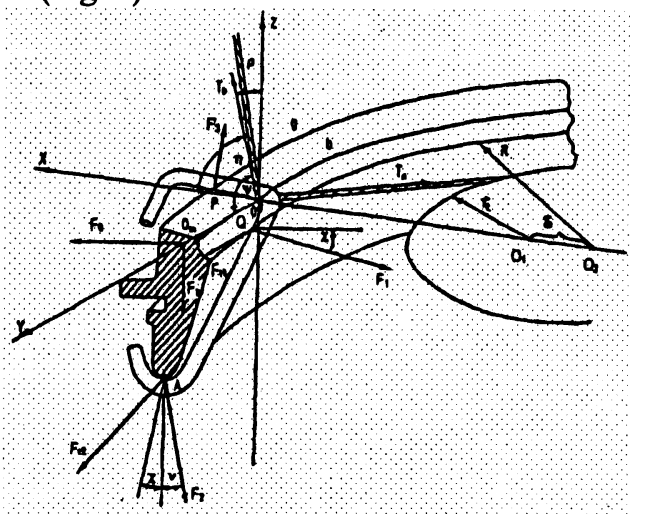

Fig. 1. Distribution of forces acting on traveller as it is moving around taper ring if there is 'malalignment' of the spindle

At the first stage of analysis the fault was assumed to be regular in nature, i.e. that the axis of the spindle deflects from the vertical so that it traces in space a geometrical figure whose cross-section is an ellipse of an elliptically factor $\delta_{x y}$. The elliptically factor $\delta_{x y}$ represents here the ratio of the ellipse greater half-axis $\delta_{x}$ to the smaller half-axis $\delta_{y}$. In a ge neral case at a time $t=0$ the half-axes of the ellipse are inclined to the co-ordinates $x$ and $y$ by an angle $\beta$. The dynamics of TRY in the discussed case is within the limits of classical mechanics.

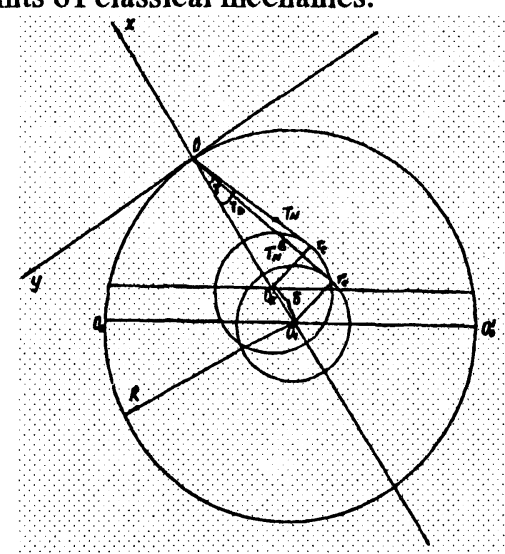

Fig.2. View of system traveller-ring-yarn (TRY) in ring plane, together with co-ordinates $x$ and $y$, at any moment of package formation

$T_{n} \quad$ - winding force in faultless operation of the system;

$T_{n}^{b}$ - winding force in 'whipping' of spindle;

Point $O$ represents a momentary position of the traveller;

Point $\mathrm{O}_{0}$ represents position of the traveller at $t=0$. 
The model of traveller motion, however, has been developed on the basis of an earlier method evolved for the purposes of analysis of the effect of spindle axis eccentricity on the dynamics of the twisting system.

The forces acting on the traveller of a weight $M$ were determined from the conditions of balanced position of the traveller on the taper ring at any moment of package formation on the bobbin. Introduced was a system of Cartesian co-ordinates $(x, y, z)$ originating at point $O$ of traveller contact with the yarn (Fig. 1) and movin g together with the traveller along the co-ordinates $x$ and $y$ in the ring plane (Fig. 2). The co-ordinate $x$ passes through the centre of the ring $O_{1}$ and the co-ordinate $y$ is tangent to the circle described by the point $O$.

As it was shown in the earlier studies [7,9] that the traveller may contact the taper ring either at two or three points, depending on the dynamic condition of the twisting system.

In the analysed case, a three -point contact of the traveller with the ring races was a ssumed as one that is more general and the most frequently occurring in TRY, while a two-point contact can be regarded as a special case.

The following symbols are used in Figs. 1 and 2:

- $R$ - ring radius;

- $r_{t}$ - momentary radius of winding of yarn onto bobbin;

- $\varphi$ - angular path of radius vector passing through points $\mathrm{O}_{1} \mathrm{O}$

- $\alpha$ - angular path of radius vector passing through points $\mathrm{O}_{2} \mathrm{O}$;

- $\mu$ - coefficient of friction between traveller and ring;

- $\mu_{n}$ - coefficient of friction between traveller and yarn;

- $g$ - acceleration of gravity;

- $\gamma, \gamma_{b}$ - angle of winding of yarn onto bobbin;

- $\boldsymbol{V}, \boldsymbol{K}$ - angles made by lines normal to ring races, directions $x$ and $y$;

- $\eta, \psi, \rho$ - angles made by 'ballooning' yam with co-ordinates $x, y$ and $z$;

- $\lambda$ - momentary angle of inclination of traveller on ring (For three-point contact this angle takes a boundary value, $\lambda_{g}$, dependent only on geometry of traveller and ring);

- $K$ - coefficient dependent on $\mu_{n}$ and angle of wrap of yarn around traveller;

- $\omega$ - spindle rotation frequency.

The traveller is acted upon by the following forces:

- $T_{N}$ - traveller driving force or yarn winding tension;

- $T_{B}$ - force with which yarn reacts to traveller;

- $F_{o}$ - centrifugal force;

- $F_{A}$ - d'Alambert force;

- $F_{g}$ - force of gravity of traveller;
- $F_{1}$ - reaction of ring to traveller at contact point $S_{1}$;

- $F_{2}$ - reaction of ring to traveller at contact point $S_{2}$;

- $F_{3}$-reaction of ring to traveller at contact point $S_{3}$;

$F_{t 1}, F_{t 2} F_{t 3}$ - forces of friction between traveller and ring at points $S_{1}, S_{2}, S_{3}$, respectively;

where:

$$
\begin{aligned}
& F_{g}=M g ; \\
& T_{N}=K \cdot T_{B} ; \\
& F_{o}=M R\left(\frac{d \varphi}{d t}\right)^{2} ; \\
& F_{A}=M R \frac{d^{2} \varphi}{d t^{2}} ; \\
& F_{t 1}=\mu F_{1} ; \\
& F_{t 2}=\mu F_{2} ; \\
& F_{t 3}=\mu F_{3} ; \\
& K=e^{\mu_{n}(\pi-\gamma-\rho)} \text { or } \quad K=e^{\mu_{n}\left(\pi-\gamma_{b}-\rho\right)} .
\end{aligned}
$$

Introduced in this work coefficient $\mu_{n}$ is a correction yarn-traveller friction coefficient, it takes into account the geometry of yarn and traveller when it is assumed that the yarn is the viscoelasticic medium [11].

To simplify calculation it is assumed, in the above equations, that the centre of gravity of the traveller is removed from the centre of the ring by a distance equal to the ring radius $R$ (Fig. 1 ).

\subsubsection{Conditions for traveller balance on the ring}

Projections, on the co-ordinates $x, y, z$, of the forces acting on the traveller give the following equations:

$$
\begin{aligned}
& T_{B} \cos \eta-T_{n} \cos \gamma+F_{o}-F_{1} \cos \kappa-F_{2} \sin v=0 \\
& T_{N} \sin \gamma-T_{B} \cos \psi-F_{t 1}-F_{t 2}-F_{A}=0 \\
& T_{B} \cos \rho-F_{1} \sin \kappa-F_{2} \cos \nu+F_{3} \cos \nu-F_{g}=0
\end{aligned}
$$

For a case when the traveller is in contact with the ring races at three points, $A, Q$, and $\mathrm{P}$, (Fig. 1) substituted into the set of equations (1) is an equation for the moments of forces acting on the travel ler at the contact point A (Fig. 1).

Fig. 3 illustrates inclination of the traveller on the ring. Marked are here a momentary inclination of the traveller by an angle $\lambda$, at two-point contact, the boundary value $\lambda_{g}$ of the inclination angle at three-point contact, and the characteristic lengths, $h$ and $\sigma$, as well as the arms, $l$ and $l_{0}$, of the moments of the forces acting on the traveller:

$$
\begin{aligned}
& T_{n} l \cos \gamma(\varphi(\alpha)) \sin \kappa+T_{n} l \sin \gamma(\varphi(\alpha)) \cos \lambda_{g}-T_{b} l \cos \eta \cos \kappa \\
& -T_{b} l \cos \psi \cos \lambda_{g}-T_{b} l \cos \rho \cos \kappa \sin \lambda_{g}+F_{g} l_{0} \cos \kappa \sin \lambda_{g} \\
& -\mu F_{1}(h-\sigma) \cos \lambda_{g}-\mu F_{3} l \cos \lambda_{g}-F_{o} l_{0} \sin \kappa-F_{A} l_{0} \cos \lambda=0 .
\end{aligned}
$$




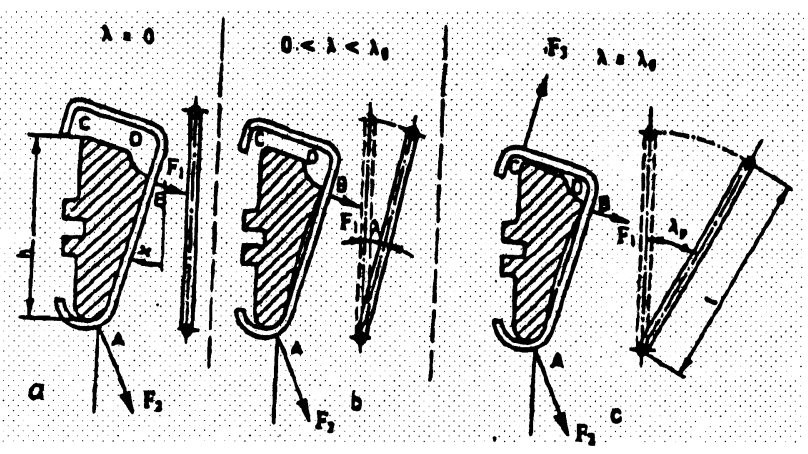

Fig. 3. Position of traveller on ring in operation a - vertical;

b-inclined by angle $\lambda$ - two-point contact; $\mathrm{c}$-inclined by angle $\lambda_{g}$ - three-point contact.

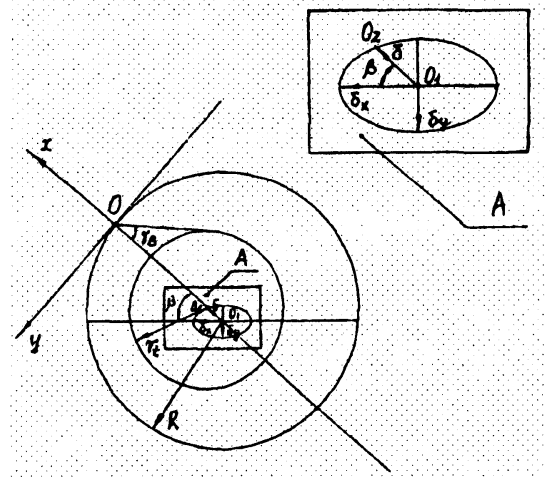

Fig. 4. Spindle axis describing an ellipse having half-axes $\delta_{x}$ and $\delta_{y}$ and inclined to $O_{0} \cdot O_{0}^{\prime}$ by angle $\beta$

\subsubsection{Reactions of the ring to traveller and tension in the} yarn

Upon transformation, the equations (1) and (2) give the reactions of the ring to the traveller and the tension in the yarn:

$$
\begin{aligned}
F_{1} & =T_{N}\left(d+\frac{m e}{n}\right)+F_{g}\left(\frac{p e}{n}-f\right) \\
F_{2} & =T_{N}\left(\frac{a}{b}-\frac{m c}{n b}\right)-F_{g}\left(\frac{p c}{n b}-\frac{\mu}{b}\right) \\
F_{3} & =T_{N} \frac{m}{n}+F_{g} \frac{p}{n}, \\
T_{N} & =\left(F_{g}\left[\cos \kappa\left(\frac{p}{n} e-f\right)-\sin v\left(\frac{p c}{n b}-\frac{\mu}{b}-\frac{p}{n}\right)\right]-F\right) \\
& \quad\left(\frac{1}{K} \cos \eta-\cos \gamma-\cos \kappa\left(d+\frac{m e}{n}\right)-\sin v\left(\frac{a}{b}+\frac{m}{n}-\frac{m c}{n b}\right)\right)
\end{aligned}
$$

The following symbols have been introduced to simplify transformation:

$$
\begin{aligned}
& a=\sin \kappa \sin \gamma-\frac{1}{K} \cos \psi \sin \kappa-\frac{\mu}{K} \cos \rho, \\
& b=\mu(\sin \kappa-\cos v),
\end{aligned}
$$

$$
\begin{aligned}
c & =\mu(\sin \kappa+\cos v) \\
d & =\frac{\cos \rho}{K \sin \kappa}-\frac{a \cos v}{b \sin \kappa} \\
e & =\frac{\cos v}{\sin \kappa}+\frac{c \cos v}{b \sin \kappa}, \\
f & =\frac{\mu \cos v}{b \sin \kappa}+\frac{1}{\sin \kappa}, \\
m & =l \cos \gamma(\varphi(\alpha)) \sin \kappa+l \sin \gamma(\varphi(\alpha)) \cos \lambda_{g} \\
& -\frac{l}{K} \cos \eta \sin \kappa-\frac{l}{K} \cos \psi \cos \lambda_{g} \\
& -\frac{l}{k} \cos \rho \cos \kappa \sin \lambda_{g}-d \mu(h-\sigma) \cos \lambda_{g},
\end{aligned}
$$

The equations (3) show that the reactions of the traveller to the ring and the winding te nsion in the yarn are dependent on the winding angle $\gamma$.

\subsubsection{Momentary yarn-onto-bobbin winding angle}

If there is dynamic 'malalignement' of the spindle (spindle whipping), the winding a ngle $\gamma_{b}$ is not equal to the winding angle $\gamma$ when the twisting-and-winding system is operating faultlessly.

In Fig. 2 is presented the plane of the ring at any moment of operation of the twisting system. The angles that the radii $O O_{1}$ and $O O_{2}$ make with the co-ordinates $\mathrm{x}$ and $y$ and with the yarn are respectively marked $\varphi$ and $\alpha$. The broken line represents momentary winding diameter when the spindle is not 'malaligned'. Assuming that spindle axis is rotating at a speed $\frac{d \varphi}{d t}=$ const. equal to traveller speed $\frac{d \alpha}{d t}=$ const., the angular path of the radius vector $O O_{1}$ will be equal to angular speed of the radius vector $O \mathrm{O}_{2}$, i.e. $\varphi=\alpha$. If rotary speed is represented by the symbol $\omega$, then:

$$
\frac{d a}{d t}=\frac{d \varphi}{d t}=\text { const. }=\omega
$$

If spindle axis, during one lap of the traveller, describes an ellipse of which the half-axes are, in time $t=0$, inclined to the co-ordinates $x$ and $y$ by an angle $\beta$, then the momentary spindle deviation $\delta$ from the vertical takes the form:

$$
\begin{aligned}
\delta & =\sqrt{1 /\left(\frac{\cos ^{2}(\varphi+\beta)}{\delta_{x}^{2}}+\frac{\sin ^{2}(\varphi+\beta)}{\delta_{y}^{2}}\right)} \\
& =\delta_{y} / \sqrt{1-\sin ^{2}(\varphi+\beta) \sqrt{\left.\delta_{y} / \delta_{x}\right)^{2}+\sin ^{2}(\varphi+\beta)}}
\end{aligned}
$$

and the winding angle $\gamma_{b}$ is expressed by the following function deriving from geometry of the system: 


$$
\begin{aligned}
\gamma_{b} & =\arcsin \frac{r_{t}}{R-\delta} \\
& =\arcsin \frac{r_{t}}{R-\frac{\delta_{y}}{\sqrt{\left[1-\sin ^{2}(\varphi+\beta)\right]\left(\frac{\delta_{y}}{\delta_{x}}\right)^{2}+\sin ^{2}(\varphi+\beta)}}}
\end{aligned}
$$

If the half-axes of the ellipse are (in time $t=0$ ) parallel to the co-ordinates $x$ and $y$, the momentary deviation of the spindle axis from the vertical takes the form:

$$
\begin{aligned}
\delta & =\sqrt{1 /\left(\frac{\cos ^{2} \varphi}{\delta_{x}^{2}}+\frac{\sin ^{2} \varphi}{\delta_{y}^{2}}\right)} \\
& =\frac{\delta_{y}}{\sqrt{1-\sin ^{2} \varphi\left(\delta_{y} / \delta_{x}\right)^{2}+\sin ^{2} \varphi}}
\end{aligned}
$$

and the winding angle $\gamma_{b}$ is expressed by the formula:

$$
\gamma_{b}=\arcsin \frac{r_{t}}{R-\delta}=\arcsin \frac{r_{t}}{R-\frac{\delta_{y}}{\sqrt{\left[1-\sin ^{2} \varphi\right]\left(\frac{\delta_{y}}{\delta_{x}}\right)^{2}+\sin ^{2} \varphi}}}
$$

As it follows from equations (5), (6) and (7) the winding angle $\gamma_{b}$ varies during one lap of the traveller and, therefore, variable also are, depending on the value the elliptically factor $\delta_{x y}$, the momentary reactions of the traveller to the ring and the momentary values of tension in the yarn. As the value of $\delta$ increases with increasing diameter of yarn package on the bobbin, so increase the amplitudes of the momentary reactions of the ring to the traveller and amplitudes of tension in the yarn.

If during one lap of the traveller the spindle axis describes a circle, i.e. if $\delta=$ const., also the winding angle is constant:

$$
\gamma_{b}=\arcsin \frac{r_{t}}{R-\delta}=\text { const. }
$$

If there are no other faults in the twisting syste $m$, constant also are - during one lap of the traveller - the reactions of the ring to traveller and the tension in the yarn. The values of the forces calculated from the equations (3) are other than in the case of faultless operation of the twisting system. These differences increase with time as the package is being formed.

\subsection{Graphical interpretation of the results for conditions of traveller balance on the ring}

Since the analytic form of the equations (3) which describe the forces acting on the traveller in its motion around the ring is rather complicated, they are presented also in graphical form.

The graphical interpretation of the analytic results regarding the conditions of traveller equilibrium on the ring has been developed taking as an example a TRY in which the ring radius $R=0.035 \mathrm{~m}$, traveller weight $m=0.000125$ $\mathrm{kg}$, momentary winding radius $r_{t}=0.0175 \mathrm{~m}$, traveller rotation frequency $f=1501 / \mathrm{s}$, coefficient of friction between traveller and ring $\mu=0.43$, and coefficient of friction between traveller and yarn $\mu_{n}=0.5$.

The angles $(\eta, \psi, \rho)$ by which the vector $T_{b}$ (reaction of 'ballooning' yarn to traveller) is oriented in respect of the Cartesian co-ordinates $x, y, z$ have approximately constant values, namely $\rho=10^{\circ}, \psi=85^{\circ}, \eta=81.5^{\circ}$.

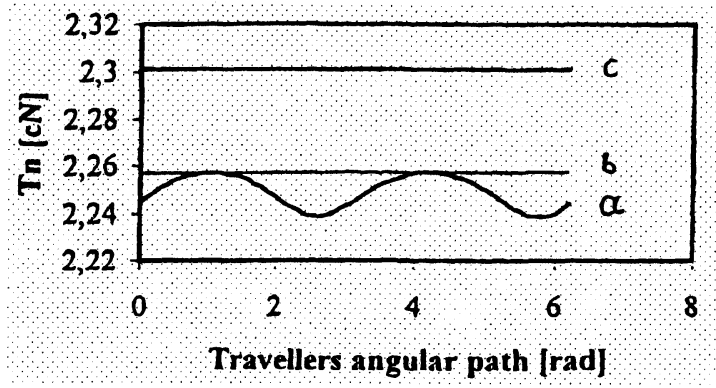

Fig. 5. Winding force $T_{n}$ for cases:

a) spindle axis describing, during one lap of the traveller, an ellipse whose half-axes are - during $t=0$ - inclined to the co-ordinates $x$ and $y$ by an angle $\beta=\frac{\pi}{6}$, the elipticity factor $\delta_{x y}=1,4285$ and $\delta_{x}=0.003 \mathrm{~m}$;

b) spindle axis describing, during one lap of the traveller, a circle of radius $\delta=0.003 \mathrm{~m}$;

c) faultless operation

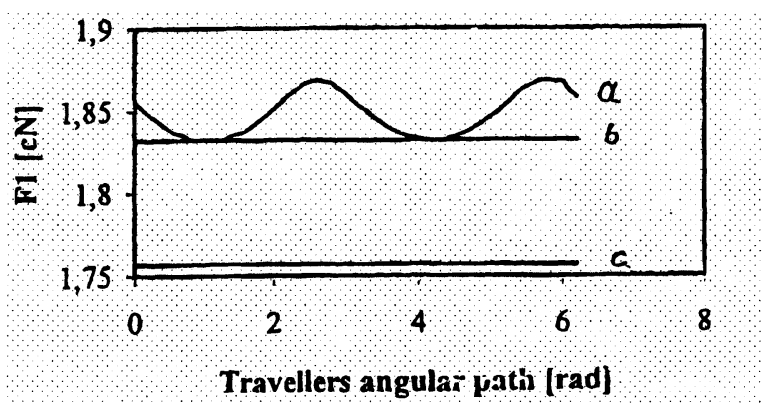

Fig. 6. Reaction $F_{1}$ for cases:

a) spindle axis describing, during one lap of the traveller, an ellipse whose half-axes are - during $t=0$ - inclined to the co-ordinates $x$ and $y$ by an angle $\beta=\pi / 6$, the elipticity factor $\delta_{x y}=1,4285$ and $\delta_{x}=0.003 \mathrm{~m}$;

b) spindle axis describing, during one lap of the traveller, a circle of radius $\delta=0.003 \mathrm{~m}$;

c) faultless operation.

In Fig. 5 curves of yam tension are presented for cases: 
(a) when spindle axis during one lap of traveller describes an elipse whose half-axes during a period $t=0$ are inclined to the co-ordinates $x$ and $y$ by an angle $\beta=\pi / 6$, the elipticity factor $\delta_{x y}=1,4285$ and $\delta_{x}=0.003 \mathrm{~m}$; (b) when spindle axis describes a circle of radius $\delta=0.003 \mathrm{~m}$; and (c) when operation is faultless.

In Fig. 6 is presented the ring-to-traveller reaction $F_{1}$, in Fig. 7 is presented reaction $F_{2}$, and in Fig. 8 is presented reaction $F_{3}$, for 'malalignment' the same as in Fig. 5.

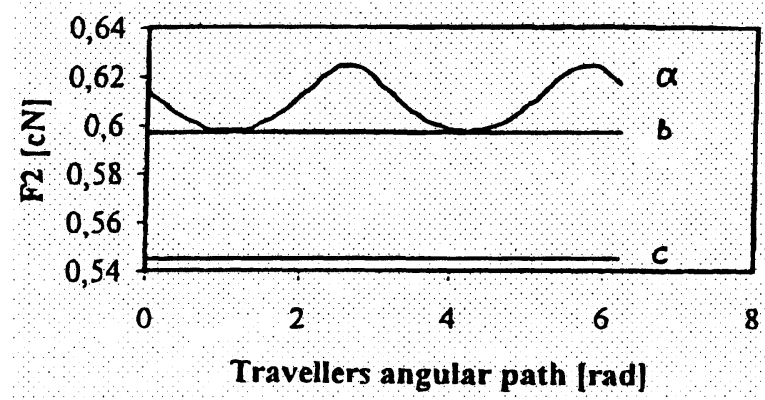

Fig. 7. Reaction $F_{2}$ for cases:

a) spindle axis describing, during one lap of the traveller, an ellipse whose half-axes are - during $t=0$ -inclined to the co-ordinates $x$ and $y$ by an angle $\beta=\pi / 6$, the elipticity factor $\delta_{x y}=1,4285$ and $\delta_{x}=0.003 \mathrm{~m}$;

b) spindle axis describing, during one lap of the traveller, a circle of radius $\delta=0.003 \mathrm{~m}$;

c) faultless operation.

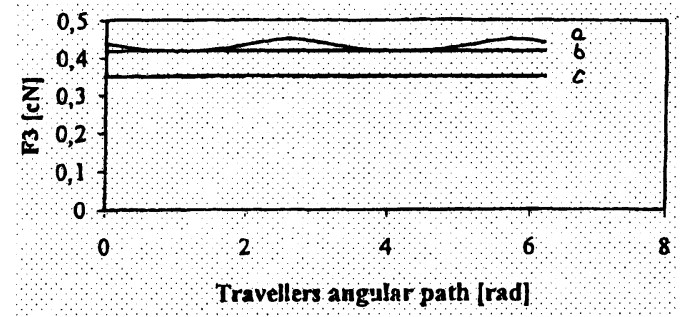

Fig. 8. Reaction $F_{3}$ for cases:

a) spindle axis describing, during one lap of the traveller, an ellipse whose half-axes are - during $t=0$-inclined to the co-ordinates $x$ and $y$ by an angle $\beta=\pi / 6$, the elipticity factor $\delta_{x y}=1,4285$ and $\delta_{x}=0.003 \mathrm{~m}$;

b) spindle axis describing, during one lap of the traveller, a circle of radius $\delta=0.003 \mathrm{~m}$;

c) faultless operation.

\section{Conclusion}

The equations (3), which describe the reactions $F_{1}$, $F_{2}$, and $F_{3}$ of the ring to traveller and the winding tension
$T_{n}$ in the yarn between the traveller and the bobbin and the graphs of the forces (Figs. 5-8) show that 'malalignment' of the spindle results in a significant increase in the values of the force $F_{1}$ and forces $F_{2}$ and $F_{3}$ (pressure of the traveller on the ring) while the force $T_{n}$ is decreased. The forces $F_{2}$ and $F_{3}$ are about three times as low as the reaction $F_{1}$ throughout the entire interval of their variation.

The increase of the force $F_{1}$, the action of which on the traveller is the most intensive, has a most destructive influence on the elements of TRY, leading to rapid deterioration of performance of the system. Such relations between the forces are in a case of three-contact inter-action between traveller with ring, the relations are changed in case of two-contact inter-action.

The results of these investigations give a new element to generating theory of twisting systems dynamic. These investigations have a big significance while the question of dynamic malalignment of spindle had not been described theoretically up to present. The known experimental investigations, for example [12], show that the problem is very important.

\section{References}

[1] P.F.Grishin: „Balloon Control”. Pla tt's Bulletin, 1954, No $6,8,11$.

[2] M.Stasiak: „Dynamika procesu przêdzenia w uk $\mathbf{u}^{3} a d z i e$ skrêtowym przêdzarki obr ${ }^{1}$ czkowej" (The Dynamics of Spinning Process in the Twisting System of a Ring Frame). Typescript, S.W.P., 1981.

[3] S.K.Batra, T.K.Grosh, M.I.Zeidman: „An Integrated Approach to Dynamic Analysis of the Ring Spinning Process, Parts I, II, IV. Textile Research Journal, 1989, No 6, 7, 1995 No 7.

[4] S.K.Batra, T.K.Grosh, M.I.Zeidman: „An Integrated Approach to Dynamic Analysis of the Ring Spinning Process, Part III. Textile Praxis International, 1992, No 9.

[5] M.Michalak, M.Stasiak: „Wzajemnie niszcz ${ }^{1}$ ce dzia ${ }^{3} a n i e$ nitki-biegacza-obr ${ }^{1}$ czki w badaniach zjawisk cieplnych uk ${ }^{3}$ adu skrêtowego przêdzarki obr ${ }^{1}$ czkowej" Cz.I, Cz.II, Cz.IIIa i b. (Reciprocally Destructive Action of Yarn, Traveller, and Ring Revealed by Analysis of the Thermal Phenomena of the Ring-Frame Twisting System). Przegl ${ }^{1} \mathrm{~d}^{\mathrm{W}} \mathrm{W}^{3} k i e n n i c z y . ~ 1993$, Nos. 5 \& 6, 1994, Nos. 10 \& 12.

[6] M.Michalak, M.Stasiak: „Interakcje w trójcz ${ }^{3}$ onowym skojarzeniu ciernym: biegacz-nitka-obr ${ }^{1}$ czka w przêdzarce obr ${ }^{1}$ czkowej we ${ }^{3}$ niarskiej" (Interaction in the Three-Element Friction System Traveller-Yarn-Ring in the Worsted Spinning Ring Frame).Proceedings of International Sympozium 'The Dynamics of Changes in the Fibre Stream in Spinning', £ód Ÿ1994.

[7] M.Michalak, M.Stasiak: Tribology of Three-Element: Traveller-Ring-Yarn Friction Arragements in Ring Spinning. Reaction of Ring to Traveller in 
Two-Contact Configuration. Part I, II. Fibres \& Textiles in Eastern Europe. 1996 No. 4, No. 2(13), 28-33, No. 3-4 (14-15), 59-61.

[8] M.Michalak, M.Stasiak: „Uwagi ogólne do dynamicznych warunków docierania obr ${ }^{1}$ czek przêdzarkowych" (General Remarks on Running-in of Spinning Rings). $2^{\text {nd }}$ International Conference 'The Dynamics of Changes in the Fibre Stream in Spinning', 1996.

[9] M.Michalak, M.Stasiak: „Tribology of Three-Element: Traveller-Ring-Yarn Friction Arragements in Ring Spinning. Part III. Reaction of Ring to Traveller in Three-Contact Configuration. Fibres \& Textiles in Eastern Europe. 1997, 5, No. 3 (18), 42-45.

[10] M.Stasiak, M.Michalak. „The Problem of Empirical
Adequancy of a Model of teh Friction Trio TRY (Traveller-Ring-Yarn) under Conditions of Spindle Eccentricity on a Ring Wool-Spinning Frame. Fibres \& Textiles in Eastern Europe. 1998, 6, nr 1 (20), 28-31.

[11] K.Kowalski. Identification of Dynamic Tension in Yam in Weft Knitting, using Co mputer Simulation and Digital Measurement, Zeszyty Naukowe Politechniki £ódzkiej, £ódŸ, 1991, No.63.

[12] J.Koritysskij, H.Lebedeva, About spindel velocity

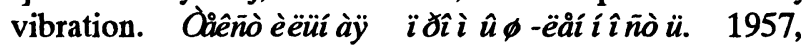
No.3. 